\title{
INCORPORACIÓN LEGAL DE LA CONSEJERÍA MATRIMONIAL OBLIGATORIA PREVIA AL DIVORCIO EN EL PERÚ
}

\author{
LEGAL INCORPORATION OF MANDATORY MARRIAGE \\ COUNSELING PRIOR TO DIVORCE IN PERU
}

Rocío del Pilar Eyzaguirre Rivas de Larios ${ }^{1}$

\section{Resumen:}

La presente comunicación tiene por objetivo determinar la idoneidad e importancia de incorporar legalmente la Consejería Matrimonial Obligatoria como requisito previo al trámite de divorcio en Perú. Para ello es necesario definir la situación del divorcio en el país, sobre la base de los reportes estadísticos publicados por las entidades oficiales y la tendencia legislativa durante las últimas décadas respecto a las figuras jurídicas relativas a la separación y disolución del vínculo matrimonial; señalaremos también las consecuencias que la ruptura produce en la vida de los cónyuges, sus hijos, el Estado y la sociedad en su conjunto. Seguidamente, analizaremos la figura de la Consejería Matrimonial Obligatoria previa, como un mecanismo de ayuda para las parejas en crisis; detallando sus efectos positivos y exponiendo los criterios que avalan su incorporación en nuestro ordenamiento legal.

Palabras clave: divorcio, crisis matrimonial, Consejería Matrimonial Obligatoria

\begin{abstract}
:
This document aims to determine the suitability and importance of incorporate legally Mandatory Marriage Counseling as a prerequisite to the process of divorce in Peru. For this reason it is necessary to define the situation of divorce in the country, on the basis of statistical reports published by official entities and the legislative trend during recent decades regarding the legal figures relating to the separation and dissolution of the marriage; We will also indicate the result that a breakup generate in the life of the spouses, their children and the state and society as a whole. Finally, we'll look at the figure of the previous mandatory marriage counseling, as a mechanism of support for couples in crisis; detailing its positive effects and exposing the criteria that guarantee its incorporation into our legal system.
\end{abstract}

Keywords: divorce, matrimonial crisis, Mandatory Marriage Counseling

Abogada por la Universidad Femenina del Sagrado Corazón - UNIFÉ, especialista en Derecho Civil. Estudios de Maestría de Persona, Matrimonioy Familia en la Universidad Católica Santo Toribio de Mogrovejo. Consejero Familiar. 


\section{INTRODUCCIÓN}

A lo largo de los últimos años se ha producido en nuestro país un incremento en el número de divorcios inscritos ante las entidades competentes para registrar tales actos, como son el Registro Nacional de Identificación y Estado Civil (RENIEC) y la Superintendencia Nacional de los Registros Públicos (SUNARP). A pesar que la Constitución Política del Perú regula el principio de protección a la familia, en la práctica ninguna autoridad o institución gubernamental ha planteado ni implementado mecanismos concretos que permitan revertir el índice de rupturas en el país; sin embargo, experiencias desarrolladas por algunas organizaciones e instituciones privadas nos vienen demostrando que si las parejas reciben orientación conyugal y familiar de manera oportuna estarán en condiciones de superar sus crisis y continuar juntos

Por lo expuesto, resulta necesario analizar la importancia de que se implementen en nuestro país, mecanismos legales y sociales destinados a prevenir y evitar las rupturas matrimoniales; ante lo cual se presenta el siguiente problema: ¿Cómo se planteará la incorporación legal de la Consejería Matrimonial Obligatoria previa al divorcio en el Perú?

Para responder a este interrogante, establecemos como objetivo de esta comunicación el proponer la incorporación legal de la Consejería Matrimonial Obligatoria previa al divorcio en el Perú, como un mecanismo de ayuda para las parejas que se encuentran atravesando una crisis; contribuyendo de este modo a disminuir el índice de las rupturas matrimoniales, en beneficio de los propios cónyuges, sus hijos y la sociedad en su conjunto.

\section{DISEÑO METODOLÓGICO}

La investigación es de tipo cualitativa, correspondiente al área de las humanidades, que aplica la descripción e interpretación como procedimientos y analiza el desenvolvimiento de la institución matrimonial en nuestro país y las dificultades por las que viene atravesando, tanto a nivel legal como social, haciendo énfasis en la importancia de la Consejería Matrimonial Obligatoria. El abordaje metodológico ha permitido recabar la información necesaria de la bibliografía obtenida en bibliotecas universitarias y virtuales, librerías especializadas y repositorios de tesis. Los sujetos de la investigación son los cónyuges que atraviesan crisis matrimoniales en el Perú. Las principales técnicas empleadas han sido la observación de documentos, el fichaje y el análisis normativo. Los instrumentos utilizados han sido las fichas de recolección de datos bibliográficas, de resumen, de normas legales y de análisis y comentarios de documentos. 


\section{ANÁLISIS Y DISCUSIÓN DE LOS RESULTADOS}

A continuación desarrollamos la investigación definiendo la situación actual del divorcio en el Perú, analizamos la figura de la Consejería Matrimonial Obligatoria previa, como un mecanismo de ayuda para parejas en crisis y explicamos las razones por las cuales la implementación de esta figura contribuiría a la disminución del índice de divorcios en nuestro país.

\section{EL DIVORCIO EN EL PERÚ.}

Entre el año 2011 y el primer semestre del año 2017 el número de divorcios inscritos ante las oficinas de RENIEC prácticamente se ha triplicado, ya que pasaron de 5,625 a 15,109 según los informes oficiales del Instituto Nacional de Estadística e Informática (INEI), publicados en diciembre del 2016 y 2017 respectivamente. Esta es una realidad que está afectando a las parejas, sin distinción de edad, estrato social, religión o lugar de residencia, ya que los departamentos en los que se inscribieron más divorcios durante el año pasado fueron Lima (4,880), Arequipa (649), La Libertad (641), Lambayeque (524), Piura (314), Junín (199), Ica (193), Cusco (169), Áncash (131) y Cajamarca (119), de acuerdo a la información proporcionada en enero de este año por la Oficina General de Comunicaciones de la Superintendencia Nacional de los Registros Públicos (SUNARP).

\subsection{Tendencia normativa relativa al divorcio en el Perú.}

La realidad descrita en el acápite anterior debe ser analizada a la luz de la tendencia normativa desarrollada por los legisladores nacionales en torno a la figura jurídica del divorcio; por cuanto su regulación ha ido variando considerablemente desde que se produjera su incorporación en nuestra legislación el siglo pasado durante el gobierno de Luis Miguel Sánchez Cerro. Efectivamente, el divorcio absoluto se incorporó en el Perú mediante la Ley de Divorcio y Matrimonio Civil Obligatorio (Decreto Ley $N^{\circ} 6889$ del 04 de octubre de 1930) y su reglamento (Ley $\mathrm{N}^{\circ} 6890$ del 08 de octubre del mismo año), la cual estableció las causales por las que el divorcio producía los mismos efectos que la nulidad (Calisaya Márquez, Angel, 2016 p. 17); posteriormente, la Ley $\mathrm{N}^{\circ} 7893$ del 22 de mayo de 1934, incorporó tres nuevas causales de divorcio, entre las que se encontraba la de mutuo disenso ${ }^{2}$ y la Ley $\mathrm{N}^{\circ} 7894$ estableció los requisitos que debían cumplir los cónyuges para poder invocar esta causal: contar con la mayoría de edad y tener, como mínimo, tres años de casados ${ }^{3}$.

CONGRESO CONSTITUYENTE DE 1931. Ley N 7893, 2017 [ubicado el 21.VII 2018]. Obtenido en https://peru. justia.com/federales/leyes/7893-may-22-1934/gdoc/

CONGRESO CONSTITUYENTE DE 1931. Ley Nº 7894, 2017 [ubicado el 21.VII 2018]. Obtenido en https://peru. justia.com/federales/leyes/7894-may-22-1934/gdoc/ 
Dos años después, el Código Civil de 1936 reguló la figura del divorcio dentro del Libro II, titulado "Del Derecho de Familia", en los artículos 247 al 291; específicamente, en el artículo 247 se detallaron las causales de divorcio (incluyendo el mutuo disenso) y en el artículo 253 se estableció que el divorcio producía la disolución del vínculo matrimonial ${ }^{4}$. Casi medio siglo después, el Código Civil de 1984 reguló en el Título IV de la Sección II del Libro III todo lo relativo al decaimiento y disolución del vínculo matrimonial, desde el artículo 332 hasta el 360, manteniéndose la regulación del divorcio absoluto en el artículo 348. A inicios de este siglo, la Ley $\mathrm{N}^{\circ} 27495$ del 07 de julio de 2001, incorporó la separación de hecho como causal de separación de cuerpos y subsecuente divorcio, eliminando la prohibición de alegar hecho propio en este caso; por lo que al haberse incluido el inciso 12 en el artículo 333 del Código Civil "cualquiera de los cónyuges puede invocar haber sido él o ella el autor/a de tal separación de hecho, esto es su propia conducta" (Cornejo Fava, María Teresa, 2014, p.29). Hace una década, la Ley N² 29227, publicada el 15 de mayo de 2008, reguló el Procedimiento No Contencioso de la Separación Convencional y Divorcio Ulterior en las Municipalidades y Notarías, a través del cual se abrió el camino para que los cónyuges que tienen dos años de casados o más, puedan solicitar ante las instancias acreditadas, la declaración de la separación convencional y luego de dos meses de producida ésta, solicitar también la disolución de su vínculo matrimonial.

\subsection{Consecuencias del divorcio.}

Esta tendencia normativa está generando en nuestra sociedad que las parejas vean al divorcio como la única o más rápida solución a sus problemas conyugales y familiares; sin advertirles sobre las consecuencias que toda ruptura produce en la vida de los cónyuges, sus hijos y también para el Estado y la sociedad en su conjunto. La consecuencia legal inmediata del divorcio es la disolución del vínculo matrimonial entre marido y mujer (Art. 348 C.C.); pero también se producen otros efectos legales: extinción de la obligación alimenticia entre ellos (salvo las excepciones establecidas en el Art. 350 C.C.); fenecimiento del régimen de sociedad de gananciales (Art. 318 inciso 3 C.C.) y el cónyuge divorciado por su culpa pierde los gananciales que procedan de los bienes del otro (Art. 352 C.C.); los cónyuges pierden el derecho a heredar entre sí (Art. 353 C.C.); la mujer pierde el derecho a llevar el apellido de quien fuera su marido agregado al suyo (Art. 24 C.C.). A nivel personal los cónyuges también sufren crisis psicológicas y es por ello que durante los últimos años los profesionales se han visto en la necesidad de implementar terapias para atender a personas divorciadas que no pueden "manejar la

CÓDIGO CIVIL DE 1936, promulgado por Decreto Supremo del 20 de agosto de 1936 [ubicado el 21.VII 2018]. Obtenido en https://es.scribd.com/doc/51134898/Codigo-Civil-de-1936 
nueva situación de soledad, retorno al hogar de sus padres o, sobre todo, para manejar la situación educacional con los hijos" (Cano, Adrián; Laspra Carme, Marin Raquel y Beuzna Maribel, 2013, p. 9); asimismo, tienen que desarrollar habilidades para ayudarlas a atravesar adecuadamente el proceso de duelo que se origina por la ruptura, el cual muchas veces conlleva "depresión, angustia, pérdida de energía vital, impotencia, desilusión, decaimiento, falta de estímulos reforzantes, subestimación, alteración perceptual y atencional" (De la Parra García, Jorge, 2008, p. 25 -40)

En cuanto a los hijos, la disolución del vínculo matrimonial les afecta en diversas áreas de su vida y su desarrollo por la desintegración familiar que se origina; a largo plazo, muchas veces su proyecto de vida no llega a concretarse del todo o se ve alterado, debido a que no cuenta con las condiciones necesarias para tomar las decisiones adecuadas en cada etapa de su vida (Ortiz Castañeda, Carla, 2017 p. 44). Entre las consecuencias que se derivan de los procesos de divorcio están las cuestiones relativas a la tenencia, el régimen de visitas y la pensión de alimentos para el o los hijos procreados durante el matrimonio; temas que son muy delicados y normalmente generan situaciones tensas entre los padres que muchas veces no logran ponerse de acuerdo fácilmente respecto a la cantidad de días y los horarios para las visitas o el importe necesario para la manutención; y en otros casos, exigen de los jueces un análisis adecuado de los hechos en los que el menor presenta, por ejemplo, determinadas características de alienación parental, un trastorno no contemplado en nuestra legislación, pero que puede ser detectado con ayuda de un informe psicológico (Peña, María Irene, 2016, p. 111). Respecto a las consecuencias psicológicas y psiquiátricas que sufren los hijos tras la ruptura, puede presentarse estrés, trastorno disocial, trastorno de ansiedad de separación, trastorno de atención, depresión, entre otros; por ello en Perú, el Instituto Nacional de Salud Mental "Honorio Delgado-Hideyo Noguchi", a través de la Dirección Ejecutiva de Investigación, Docencia y Atención Especializada de Niños y Adolescentes, cuenta desde hace dos décadas con el Programa Terapéutico de Ansiedad a cargo de un equipo multidisciplinario de profesionales de la salud, que atiende, diagnostica y brinda tratamiento a niños y adolescentes que sufren trastornos de ansiedad, entre los cuales se encuentra el de ansiedad de separación; al constituir este tipo de trastornos un gran problema de salud pública en dicha población, de acuerdo a los estudios epidemiológicos de salud mental realizados por dicha institución (Instituto Nacional de Salud Mental "Honorio Delgado-Hideyo Noguchi" $\mathrm{s} / \mathrm{f}, \mathrm{p} .11)$. En cuanto a las consecuencias en el ámbito académico, una investigación tuvo como grupo de estudio a veinticinco alumnas del segundo año de secundaria de una institución educativa piurana, cuyos 
padres se encontraban separados o divorciados, concluyendo que más de la mitad (52\%) había visto afectado su rendimiento académico y su nivel de aprendizaje. (Zuazo, Norka, 2013, p. 86)

Con relación a las consecuencias que el divorcio genera para el Estado, está el coste económico debido a los programas sociales que deben implementarse para ayudar a las personas divorciadas y sus hijos, a fin de que puedan hacer frente a esta crisis. En Estados Unidos vemos que "en un estudio que incluye datos exhaustivos de los 50 Estados, los expertos estiman que el divorcio y la consiguiente crianza de los hijos por padres solos, supone para los contribuyentes al menos 112 mil millones de dólares al año" (Olesti, María José, 2012 p. 3); en Canadá, el Institute of Marriage and Family concluye que "el coste de ayudar a las familias rotas es de casi 7 mil millones de dólares al año, (...) dirigidos a facilitar la vivienda, los gastos de guardería y la asistencia social" (The Centre for Social Justice, 2009, p. 63); y en Reino Unido se estima que el costo financiero que generan las rupturas familiares para el país asciende aproximadamente a los 20-24 billones de libras esterlinas, que incluyen tanto los costos por apoyo directo a padres solos como el impacto indirecto de los divorcios en el empleo, la educación, la salud, los aspectos criminológicos y policiales, entre otros (The Center of Social Justice, 2009, p. 63). Si bien en países con economías desarrolladas, las personas pueden acceder a las ayudas sociales descritas anteriormente, no ocurre lo mismo en nuestro país porque es un aspecto que el gobierno aún no toma en cuenta, ya que sólo se orienta a desarrollar programas sociales destinados a mejorar la calidad de vida de los peruanos que viven en situación de pobreza y pobreza extrema, tales como Juntos, Cuna Más, Qali Warma, SIS, Foncodes, entre otros, previa acreditación de su situación y registro en el Sistema de Focalización de Hogares (SISFOH).

\section{LA CONSEJERÍA MATRIMONIAL OBLIGATORIA.}

La Consejería Matrimonial es un procedimiento a través del cual los cónyuges, que atraviesan por una crisis en su relación, recurren a la asesoría de un profesional especializado para que los ayude a detectar las causas del conflicto, desarrollar alternativas de solución y aprender estrategias que les permitan evitar futuros problemas (Escrivá, Javier, 2013, p. 60)

\subsection{Características y efectos de la Consejería Matrimonial Obligatoria.}

La Consejería Matrimonial Obligatoria como figura legal, es un mecanismo de solución preventivo por el cual se exige a los cónyuges que recurran a dicho procedimiento antes de dar inicio a cualquier trámite de 
separación o divorcio; ya que el consejero matrimonial "respaldándose en una formación integral de la institución matrimonial, tiene el cometido de mostrar de forma comprensible para los cónyuges el ser matrimonial, a la vez que anunciar las posibles consecuencias personales y familiares de una ruptura" (Cano, Adrián; Laspra, Carmen ; Martin, Raquel, y Beuzna, Maribel, 2015, p. 13); lo que el profesional realmente va a buscar es ayudarles a comprender que esa crisis no necesariamente tiene que derivar en la separación y disolución del vínculo y finalmente estos podrán tomar una decisión sobre la base de una evaluación completa y objetiva.

El optar por la Consejería Matrimonial Obligatoria beneficia no sólo a los cónyuges en crisis, sino también a la familia y el Estado. Respecto a los cónyuges, ayuda a que afronten el problema con objetividad, brindándoles estrategias que faciliten la comunicación, proponiendo alternativas que tomen en cuenta los intereses de ambos y de sus hijos, y mostrando con claridad las ventajas, inconvenientes y consecuencias de todas las posibles soluciones por las que puedan optar (Escrivá-Ivars, Javier, 2001, p. 132); en suma, desarrolla una labor educativa con el varón y la mujer para que puedan lograr cambios positivos en su relación conyugal (Barrera Cruz, Teresa, 2013, p.47-48). En cuanto a la familia, estimula el descubrimiento del sentido de vida familiar, animando a los cónyuges a que elaboren un proyecto familiar concreto y vivencial (Paz Alcalde, Nicolás, 2015, p. 118 -119); facilita el aprendizaje de herramientas y el desarrollo de habilidades que ayuden a resolver los conflictos, lograr una adecuada gestión de la convivencia familiar, mejorar la comunicación y la relación con los hijos (Martínez de Aguirre, Carlos, 2015). Con relación al Estado, esta figura está enmarcada dentro del principio constitucional de protección a la familia; constituye una iniciativa al servicio de esta institución, que busca afirmar su valor social y ayudar a que continúe siendo la célula básica de nuestra sociedad; (Tonini, Franca, 2011, p. 11) como recurso social a favor del matrimonio y la familia permitirá afrontar y revertir diversas situaciones críticas que hoy los aquejan y repercuten negativamente en lo familiar y colectivo.

\subsection{Criterios para una posible regulación de la Consejería Matrimonial Obligatoria.}

En nuestro país existe un vacío legal en torno a la figura de la Consejería Matrimonial Obligatoria previa al divorcio, ya que actualmente nuestro ordenamiento legal no regula ninguna figura jurídica destinada a ayudar a los cónyuges en crisis para que desistan de optar por la ruptura matrimonial. Así vemos que la Conciliación Extrajudicial es un mecanismo alternativo de resolución de conflictos que, en temas de familia, sólo considera "como materias conciliables aspectos referentes a pensión de alimentos, régimen 
de visitas, tenencia y otras que se deriven de la relación familiar y respecto de las cuales las partes tengan libre disposición" (Pérez Solf , Iván, 2013, p. 259); es decir, se ocupa de situaciones que se originan de la relación entre los padres y sus hijos, una vez producida la disolución del vínculo. En cuanto al Derecho Comparado, verificamos que la legislación española regula la mediación familiar como un método de resolución de conflictos que tiende a "facilitar la gestión del divorcio, pero limitada únicamente a evitar la conflictividad en la ruptura" (Martínez Aguirre, Carlos, 2015); es una figura jurídica que tiene como objetivo ayudar a los cónyuges que han decidido separarse, a negociar y resolver de manera pacífica sus desacuerdos respecto de las relaciones conyugales, paterno-filiales y patrimoniales, tomando en consideración las necesidades de cada uno de los miembros de la familia, especialmente de los hijos. (Escrivá-Ivars, Javier 2001, p 133)

No obstante lo expuesto, sabemos que en algunas ciudades del país se vienen desarrollando alternativas de solución concretas frente a las crisis matrimoniales. Una de ellas es el programa para varones denominado Verdadera Hombría, que se lleva a cabo en las ciudades de Arequipa, Barranca, Chincha, Huacho y Lima; el cual consiste en un fin de semana donde participan hombres casados o convivientes (sin impedimento de casarse) de entre treinta a cincuenta años, quienes en esos dos días realizan una revisión de su desempeño como esposos, padres, trabajadores y ciudadanos; y al término del mismo, la mayoría de los participantes toman la decisión de ordenar sus vidas en beneficio propio, de su matrimonio y su familia; incluso si ya estaban separados de sus esposas o tenían la intención de separarse, desisten de hacerlo y retoman su relación. También tomamos conocimiento que en agosto del año dos mil dieciséis el Instituto de Ciencias para el Matrimonio y la Familia (ICMF) de la Universidad Católica Santo Toribio de Mogrovejo (USAT), en coordinación con la Gerencia Regional de Programas Sociales del Gobierno Regional de Lambayeque, presentó un proyecto para la implementación de módulos de consejería matrimonial, donde las parejas de la jurisdicción podrían acudir a recibir ayuda desde las primeras etapas del matrimonio para la solución de sus conflictos ${ }^{5}$; sin embargo, nos informan que hasta la fecha dicho proyecto no se ha concretado. Ambas propuestas nos muestran que si las parejas reciben orientación conyugal y familiar de manera oportuna, estarán en condiciones de superar sus crisis y continuar juntos.

Por todo lo expuesto anteriormente, consideramos oportuna y necesaria la incorporación de la Consejería Matrimonial Obligatoria previa al divorcio

Cfr. INSTITUTO DE CIENCIAS PARA EL MATRIMONIO Y LA FAMILIA DE LA UNIVERSIDAD CATÓLICA SANTO TORIBIO DE MOGROVEJO. ICMF - USAT presenta proyecto a favor de la familia, 2016 [ubicado el 24.X 2017]. Obtenido en http:/ / www.usat.edu.pe/noticias/icmf-noticias/icmf-usat-presenta-proyecto-favor-de-la-familia/ 
en nuestro ordenamiento legal; en virtud a que se encuentra enmarcada dentro del principio de protección de la familia reconocido por la Constitución Política de $1993^{6}$ y el Código Civil de 19847, que la consideran un instituto fundamental de la sociedad; así como en lo dispuesto por el Acuerdo Nacional que contempla en su décimo sexta Política de Estado el fortalecimiento de la familia como espacio fundamental del desarrollo integral de las personas. Asimismo, se sustenta en el derecho que todo niño y adolescente tiene de vivir, crecer y desarrollarse en el seno de una familia, tal como lo dispone el artículo $9^{\circ}$ del Código de Niños y Adolescentes; y en la idoneidad de desarrollar formas alternativas de solución de conflictos distintas a la judicial, que además de ayudar a disminuir la sobrecarga judicial permitirá a las partes involucradas solucionar sus conflictos de una forma más adecuada para todos (Montes de Oca Vidal, Alipio, 2013, p.111). Esta incorporación permitirá que más parejas puedan acceder a este tipo de ayuda en todo el territorio nacional, contribuyendo de este modo a disminuir el índice de divorcios en el Perú, en beneficio de los propios cónyuges, sus hijos y la sociedad en su conjunto.

\section{CONCLUSIONES}

Elnúmero de divorcios inscritos en nuestro país vieneincrementándose durante los últimos años y está afectando a las parejas que atraviesan por una crisis conyugal, sin distinción de edad, estrato social, religión o lugar de residencia.

La tendencia normativa de los legisladores nacionales en torno a la figura jurídica del divorcio ha sido la de simplificarlo, dotándolo de mayor celeridad y flexibilidad para un más fácil acceso por parte de los cónyuges, quienes recurren a este procedimiento considerando que es la única solución a sus problemas sin tomar en cuenta todas las consecuencias que una decisión como ésta va a producir en sus propias vidas, la de sus hijos y también para el Estado y la sociedad en su conjunto.

La Consejería Matrimonial Obligatoria es un mecanismo de solución de conflicto de carácter preventivo que aún no está regulado en nuestro ordenamiento legal, pero cuyos efectos son beneficiosos tanto para los cónyuges en crisis, como para la familia en sí y también para el Estado.

6 La Constitución Política del Perú establece en su artículo 4: “La comunidad y el Estado (...) protegen a la familia y promueven el matrimonio. Reconocen a estos últimos como institutos naturales y fundamentales de la sociedad. La forma del matrimonio y las causas de separación y de disolución son reguladas por la ley"; y en su artículo $7^{\circ}$ : “Todos tienen derecho a la protección (...) del medio familiar (...) así como el deber de contribuir a su promoción y defensa (...)". Constitución Política del Perú, 1993 [ubicado el 30.V 2017]. Obtenido en http:/ /www4.congreso.gob. pe/ntley/Imagenes/Constitu/Cons1993.pdf

El Código Civil de 1984 establece en su artículo 233: “La regulación jurídica de la familia tiene por finalidad contribuir a su consolidación y fortalecimiento, en armonía con los principios y normas proclamados en la Constitución Política del Perú". 
El principio constitucional de protección a la familia, el derecho de todo niño y adolescente a vivir en una familia, la necesidad de implementar formas alternativas de solución de conflictos distintas a la judicial y el vacío legal existente en nuestro país respecto de mecanismos destinados a ayudar a los cónyuges en crisis; hacen oportuna y necesaria la incorporación legal de la Consejería Matrimonial Obligatoria previa al divorcio.

La incorporación legal de la Consejería Matrimonial Obligatoria previa al divorcio permitirá que más parejas puedan acceder a este tipo de ayuda en todo el territorio nacional, contribuyendo de este modo a disminuir el índice de divorcios en el Perú, en beneficio de los propios cónyuges, sus hijos y la sociedad en su conjunto.

\section{REFERENCIAS BIBLIOGRÁFICAS}

\section{Libros}

Escrivá - Ivars, Javier (2001). Matrimonio y Mediación Familiar: Principios y elementos esenciales del matrimonio para la mediación familiar, Madrid: Ediciones Rialp S.A.

Ministerio de Justicia y Derechos Humanos (2015). Código Civil: Decreto Legislativo $N^{\circ} 295,16^{\mathbf{a}}$ ed, Lima:Ministerio de Justicia y Derechos Humanos.

\section{Obra publicada por una institución}

Instituto Nacional de Estadística e Informática-INEI (2016). Perú: Natalidad, Mortalidad y Nupcialidad 2015, Lima: INEI

Instituto Nacional de Estadística e Informática ( 2017). Perú: Natalidad, Mortalidad y Nupcialidad 2016, Lima:INEI

Instituto Nacional de Salud Mental "Honorio Delgado-Hideyo Noguchi" (2010). Afronte terapéutico para niños y adolescentes con trastornos de ansiedad, Lima: INSM “Honorio Delgado-Hideyo Noguchi”

The Center for Social Justice (2009). Every family matters: an in-depth reviewof family law in Britain, Londres:The Centre for Social Justice.

The Family Watch (2013). Mecanismos de prevención frente a las crisis familiares, Madrid: TFW 


\section{Tesis}

Calisaya Márquez, Angel Alfredo (2016). La indemnización por inestabilidad económica tras la separación de hecho: criterios para la identificación del cónyuge más perjudicado, Tesis para optar el grado de Magíster en Derecho Civil, Pontificia Universidad Católica del Perú, Lima.

Ortiz Castañeda, Carla Emperatriz (2017). Derecho de los hijos a una indemnización por daño moral ocasionado por el padre/madre culpable del divorcio, Tesis para optar el Título de Abogada, Universidad de Piura, Piura.

Peña Barrientos, María Irene Elvencia (2016). El controvertido síndrome de alienación parental como patología jurídica y sus implicancias en el binomio legal tenencia - régimen de visitas en la legislación de familia, Tesis para optar el Título de Abogado, Universidad de Piura, Piura.

Zuazo Olaya, Norka Tatiana (2013). Causas de la desintegración familiar y sus consecuencias en el rendimiento escolar y conducta de las alumnas de segundo año de la Institución Educativa Nuestra Señora de Fátima de Piura, Tesis de posgrado, Universidad de Piura, Piura.

\section{Artículos de Revista}

Barrera Cruz, Teresa. (2013). Crisis familiares y mediación familiar. Mecanismos de prevención frente a las crisis familiares, Madrid, The Family Watch Instituto Internacional de Estudios sobre la Familia, pp. 45-50.

Cano, Adrián; Laspra, Carmen; Martin, Raquel y Beuzna Maribel (2013). "Mediación preventiva y terapia familiar". Mecanismos de prevención frente a las crisis familiares, Madrid, The Family Watch Instituto Internacional de Estudios sobre la Familia, p. 9-15.

Cornejo Fava, María Teresa (2014). La Separación de Hecho de los Cónyuges como causa de la Separación de Cuerpos y del Divorcio: Peculiaridades. Persona y Familia, No 03 (1), 2014, pp. 25-31.

De la Parra García, Jorge (2008). Experiencia emocional y ruptura de pareja, Familia: Revista de ciencias y orientación familiar, N 36, 2008, pp. 25-40.

Escrivá Ivars, Javier (2013). Virtudes primarias del mediador familiar: modestia y sensatez. Mecanismos de prevención frente a las crisis familiares, Madrid, The Family Watch Instituto Internacional de Estudios sobre la Familia, pp. 59-66. 
Montes de Oca Vidal, Alipio (2013). "Mecanismos alternativos de solución de conflictos", Lumen, Revista de la Facultad de Derecho de la Universidad Femenina del Sagrado Corazón, Nº 9, 2013, pp. 111-118.

Olesti, María José (2012). ¿Hay una 'nueva vida' después del divorcio? Cómo podrían evitarse muchas rupturas (y sus consecuencias), IFFD PAPERS, No 8, abril 2012, pp. 1-4.

Paz Alcalde, Nicolás (2015). La familia en busca de sentido. Una metodología para trabajar la dimensión existencial de las familias, Familia: Revista de ciencias y orientación familiar, N 51, 2015, pp. 117-132.

Perez Solf, Iván (2013). La conciliación extrajudicial en temas de familia (Ley $\mathrm{N}^{\circ} 29876$ que modifica la Ley $\mathrm{N}^{\circ} 26872$ y el D.L. 1070), IUS Revista de investigación de la Facultad de Derecho de la Universidad Católica Santo Toribio de Mogrovejo, Año III Nº, setiembre 2013, pp. 1-14.

Tonini Zacarini, Franca (2011). Origen y evolución histórica de la Orientación Familiar y de los Centros de Orientación Familiar en el siglo XX. Parte primera: los COF a nivel Internacional, Familia: Revista de ciencias y orientación familiar, $\mathrm{N}^{\circ} 42$, pp. 9-24.

Tonini Zacarini, Franca (2011). Origen y evolución histórica de la Orientación Familiar y de los Centros de Orientación Familiar en el siglo XX. Parte segunda: Orientación familiar y COF en Europa, Familia: Revista de ciencias y orientación familiar, $\mathrm{N}^{\circ} 42$, pp. 27-60.

\section{Recursos electrónicos}

Acuerdo Nacional Políticas de Estado, 2014 [ubicado el 30.IV 2017]. Recuperado de :http:/ /acuerdonacional.pe/politicas-de-estado-del-acuerdonacional/politicas-de-estado $\% \mathrm{E} 2 \% 80 \% 8 \mathrm{~B} /$ politicas-de-estado-castellano/

Código Civil de 1936, promulgado por Decreto Supremo del 20 de agosto de 1936 [ubicado el 21.VII 2018]. Recuperado de : https:/ /es.scribd. com/doc/51134898/Codigo-Civil-de-1936

Congreso Constituyente de 1931. Ley $N^{\circ}$ 7893, 2017 [ubicado el 21.VII 2018]. Recuperado de: https:/ / peru.justia.com/federales/leyes/7893-may-22-1934/gdoc/

Congreso Constituyente de 1931. Ley $N^{\circ}$ 7894, 2017 [ubicado el 21.VII 2018]. Recuperado de: https:/ / peru.justia.com/federales/leyes/7894-may-22-1934/gdoc/ 
Congreso de la República Código de los Niños y Adolescentes, 1998 [ubicado el 29.IV 2017]. Obtenido en http://www4.congreso.gob.pe/ comisiones/1998/mujer/CNA.HTM

Congreso de la República Constitución Política del Perú [ubicado el 29.IV 2017]. Recuperado de : http://www4.congreso.gob.pe/ntley/ Imagenes/Constitu/Cons1993.pdf

Martínez Aguirre, Carlos (2015). Crisis matrimoniales: de la reacción a la prevención, 2015 [ubicado el 28.VI 2017]. Recuperado de : http://www. thefamilywatch.org/wp-content/uploads/escrito-juridico-10.pdf

Superintendencia Nacional de los Registros Públicos (2018) Inscripción de divorcios a nivel nacional creció en 19 departamentos el 2017, 2018 [ubicado el 13.II 2018]. Recuperado de : https:/ / www.sunarp.gob.pe/PRENSA/inicio/ post/2018/01/17/inscripcion-de-divorcios-a-nivel-nacional-crecio-en-19departamentos-el-2017

Fecha de recepción : : 31 de agosto de 2018

Fecha de aceptación : 27 de octubre 2018 